\title{
Städtische Sozialstrukturen und residentielle Segregationsmuster am Beispiel Basel-Stadt
}

\author{
Susanne Eder, Basel
}

\section{Einleitung}

Globalisierung und Postfordismus haben in westlichen Industrienationen $\mathrm{zu}$ gesamtgesellschaftlichen Entstrukturierungstendenzen geführt. Zu beobachten sind die Auflösung von klar abgegrenzten Gesellschaftsklassen und die Bildung neuer Lebensstil- oder Milieugruppen (Dangschat 1998, Hradil 1987, Müller 1992, Vester 1993). Bei gleichzeitigem Fortbestehen traditioneller sozialer Schichtstrukturen kommt es zu einer neuen sozialen Polarisierung.

Die neuen sozialen Entwicklungen drücken sich im Stadtraum in einer neuartigen sozialräumlichen Segregation der Wohnbevölkerung aus. Durch selektive innerstädtische Migration tritt an die Stelle relativ homogener Viertelsstrukturen ein eher kleinräumig heterogenes Raumgefüge, das durch Statusunterschiede in unmittelbarer Nachbarschaft geprägt ist (Noller 1999, Borchers \& Tempel 1998, Herlyn \& HarTh 1996).

Die in vielen Studien nachgewiesene Verstärkung der v.a. ethnischen - räumlichen Absonderung oder Segregation im städtischen Raum in den 90er Jahren ist ein vieldiskutiertes Thema in Stadtpolitik und -verwaltung und wird mit der Gefahr von "Ghettobildung» in Zusammenhang gebracht (MAHNIG 2001). Bei zunehmender sozialer Marginalisierung wird die räumliche Absonderung von benachteiligten Gesellschaftsgruppen als potentielle Bedrohung für den sozialen Frieden der Stadt betrachtet. In der Annahme, dass sich die Tendenz der räumlichen Ungleichverteilung nachteilig auf die gewünschte Integration der betroffenen sozialen Gruppen auswirkt, werden Stadtviertel mit einem hohen Anteil an Migranten und sozialer Marginalisierung mit Massnahmen zur ethnisch-sozialen Durchmischung belegt (MAHNIG 2001).

Ziel dieses Beitrags ist die Erfassung von sozialräumlichen Stadtstrukturen unter dem Aspekt der selektiven Ballung bestimmter Bevölkerungsgruppen in städtischen Teilräumen. Die Verortung der Konzentrationspunkte ausgewählter Merkmalsgruppen in der Stadt ist wichtig für eine über die Beschreibung der räumlichen Ungleichverteilung hinausgehende Analyse. Sie kann Aufschluss darüber geben, für welche Stadtgebiete eine Auflösung der sozial homogenen Viertelsstrukturen beobachtet werden kann und welche Quartiere immer noch vorherrschend sozial einheitlich gegliedert sind oder für welche sich neue Konzentra- tionspunkte und damit mögliche Schwerpunktgebiete städtischer Planung abzeichnen. Ausgehend von der These, dass die Gegebenheiten des Wohnungsmarktes eine zentrale Bedeutung für die Ausbildung, Abgrenzung und Festigung von sozialen Raummustern haben (vgl. Herlyn et al. 1991, Spellerberg 1997), wird die Homogenität bzw. Heterogenität der gefundenen Sozialräume mit der vorherrschenden Art des Wohnraumangebots (wie Bebauungsstruktur, Wohnungsgrösse, Grünflächenanteil) in Verbindung gesetzt.

\section{Gesellschaftlicher Wandel und Raumzusammen- hang im Spiegel der neueren wissenschaftlichen Literatur}

Seit einigen Jahrzehnten werden weitreichende sozioökonomische Veränderungen im Zuge von Globalisierung und Postfordismus beobachtet (in der Literatur umfassend beschrieben, siehe z.B. LeFEBVRE 1991, Dangschat 1998, Soja 1995, Krätke 1991, KrätKe 1997, BaскHaus 1999). Wichtigste Charakteristika sind u.a. Internationalisierung und Flexibilisierung von Wirtschaftsaktivitäten, Rückbau des Wohlfahrtsstaates, kulturelle Homogenisierung auf internationaler Ebene bei gleichzeitig zunehmender ethnisch-kultureller Vielfalt innerhalb einzelner Städte und Regionen. Infolge der gesamtgesellschaftlichen Veränderungen ist die soziale Ordnung zunehmend komplexer strukturiert. Es vollzieht sich ein Wandel der Sozialstrukturen vom «Standardlebenszyklus» der 50er und 60er Jahre zu einer Individualisierung der Lebensführung und folglich zu einer sozioökonomischen Heterogenisierung in neue Lebensstil- oder Milieugruppen (DANGSChat 1998, Hradil 1987, Müller 1992, Noller 1999, VeSTER 1993). In der Sozialforschung wird der rasche Wandel auf der lokalen Ebene des sozialräumlichen Gefüges der Stadt als Trend der sozialen Strukturen weg von der vertikal (nach sozioökonomischer Lage) geschichteten Klassen- hin zur horizontal (nach Kriterien des Lebensstils) ausdifferenzierten Lebensstilgesellschaft gesehen (Hradil 1990, Vester 1993, Vester 1998). Kennzeichen sind soziale Fragmentierung, Auseinanderdriften sozialräumlicher Strukturen (Segregation) und der sozioökonomischen Lagen (Polarisierung) (Dangschat 1994, Hahn 1999, Friedrich 1999). Die für diese Studie relevanten gesellschaftlichen Veränderungen lassen sich nach Hradil 1999, Dangschat 1998, Borchers \& Tempel 1998, Müller 1989 u.a. wie folgt zusammenfassen:

- Haushalttyp. Zerfall der traditionellen Kernfami- 
lie: Reduzierung der Haushaltsgrösse mit Zunahme der kinderlosen (Ehe-)Paare, Zuwachs an Einpersonenhaushalten, Anstieg des Heiratsalters der Frauen bei gleichzeitiger Abnahme der Heiratsquoten sowie Anstieg der Scheidungsrate.

- Erwerbsleben.Zunahme von Doppelverdienerhaushalten, Auflösung der traditionellen Berufskategorien mit bestimmtem Sozialstatus und Milieutyp durch Entstehung neuer Berufsfelder, Auflösung der traditionellen «nivellierten» Mittelschicht der Nachkriegsjahre (SCHELSKY 1965: 332f.).

- Einkommen. Generelle Erhöhung der Durchschnittseinkommen bei gleichzeitiger Vergrösserung der Kluft zwischen Arm und Reich.

- Mobilität. Anstieg der sozialen, sozio-professionellen und räumlichen Mobilität infolge Befreiung von sozialen Verankerungsmechanismen (z.B.gelokkerte Familienverantwortung) und Bildungsreformen.

Sozialgeographen, die den Einfluss des Raums auf die Sozialstrukturen miteinbeziehen, sehen in gegebenen Raumstrukturen Produkt und Bedingung für soziale Lebensformen. Der Zusammenhang von Gesellschaft und Raum drückt sich in klar abgegrenzten «natürlichen Lebensräumen» mit Bewohnern gleichartiger Sozialcharakteristika aus, die ungleich im Stadtgebiet verteilt sind. Burgess, Park und McKenzie prägten hierfür den Begriff «residentielle Segregation» (BLasius 1988: 410).

Im neueren sozialwissenschaftlichen Diskurs geht man davon aus, dass physisch-materielle Raumstrukturen sozial modifiziert sind, so dass Gesellschaftsphänomene in der Struktur (sozial-)räumlicher Beziehungen reflektiert werden (Vertreter dieses neuen Mensch-RaumVerständnisses sind u.a. Bourdieu 1991, Lefebvre 1991 und Werlen 1995). Residentielle Segregation ist demnach die Verräumlichung sozialer Diskrepanzen im Stadtraum. Der Prozess ist in Zusammenhang mit sozioökonomischen Konkurrenzkämpfen von Bevölkerungsgruppen mit unterschiedlichen Chancen auf dem Wohnungsmarkt zu sehen, z.T. gesteuert durch die städtische Wohnungspolitik. Folge ist die Verdrängung «unterer» durch «obere» soziale Gruppen oder die auf bestimmte Gebiete konzentrierte Zuwanderung neuer Bevölkerungsgruppen. Für BouRDIEU verstärkt sich die sozialräumliche Segregation mit zunehmender Einkommensungleichheit, höherer Statusdifferenzierung oder wachsendem Anteil der (ethnischen) Minoritäten an der Gesamtbevölkerung (Bourdieu 1983).

Basierend auf der These der vertikalen Gesellschaftsgliederung als traditionelle Dimension der gesellschaftlichen Ungleichheit wurden Segregationserscheinungen bisher v.a. nach den soziodemographischen und sozioökonomischen Faktoren Nationalität, Ethnie, Religionszugehörigkeit, Einkommen, Bildung und Beruf untersucht (Blasius 1988: 411, LichtENBERGER
1998: 223). Ergebnis war, dass sich stets bestimmte Gruppen in von ihnen bevorzugten Wohngebieten durchsetzen und die übrigen Gruppen in die verbleibenden Gebiete verdrängen. Wird eine Personengruppe in ein einziges Teilgebiet verdrängt und damit räumlich isoliert, spricht man von «Ghetto». Man fand ferner heraus, dass unterschiedliche sozioökonomische und -demographische Merkmalsgruppen verschiedene städtische Raummuster bilden: die ethnische Dimension ballungsartige, die demographische zonale, zentral-periphere und die berufliche Dimension sektorielle Raumstrukturen (LiCHTENBERGER 1998: 242).

Die Veränderung der Stadtgesellschaft von der Klassen- zur Lebensstilstruktur führt zu einem neuen Sozialraumgefüge der Stadt. Die «Lehrbuchgeometrie» der zonalen und sektoriellen residentiellen Muster der Klassengesellschaft, die oft mit Viertelgrenzen korrespondieren, löst sich auf in ein Mosaik kleinräumig segregierter Lebensstilgruppen, es bilden sich sog. «soziale Inseln» (HelBrechT 1997: 11). In diesem Zusammenhang stehen auch die Konzepte der «geteilten Stadt» von Castells und Marcuse (Castells 1994, Marcuse 1995). WyLY vertritt dagegen die These, dass die sozialen und räumlichen Veränderungen va. das obere Ende der sozialen Leiter (doppelverdienende Zweipersonenhaushalte, wohlhabende Einpersonenhaushalte) betreffen, während für grosse Teile der Gesellschaft eine Segregation nach Klassen- bzw. Viertelstrukturen unverändert fortbesteht (WYLY 1999: 313).

\section{Fallstudie Basel}

\subsection{Fragestellung und Vorgehensweise}

Die vorliegende Studie versucht den sozialräumlichen Strukturwandel zu einem kleinräumig-heterogenen Mosaik sozialer Welten zu dokumentieren. Haushaltsgruppen, die hinsichtlich eines Merkmals identisch sind, werden nach ihrer Ungleichverteilung im Stadtraum untersucht. Die sozialräumliche Entmischung der Wohnbevölkerung wird mathematisch über die Formel des Segregationsindex mehrerer sozioökonomischer Variablen auf der Quartiers- und Baublockebene im Kanton Basel-Stadt nachvollzogen.

Der heutige Trend «horizontaler» Gesellschaftsverschiebungen und daraus resultierender neuer sozialer Gruppierungen kann mit den traditionellen Segregationsmerkmalen (s.o.) nicht mehr adäquat beschrieben werden (zu neuen Determinanten sozialer Ungleichheit siehe Dangschat 1994, Hradil 1999). In dieser Studie werden die Segregationsindices für drei verschiedene Variablen ermittelt, die diesen Entwicklungen Rechnung tragen: Mit der Variablen «Heimatstaat" geht ein traditioneller Segregationsfaktor in die Berechnungen ein. Die synthetische Variable «sozioprofessionelle Kategorie» korrespondiert laut SCHWEI- 
ZERISCHEM BUNDESAMT FÜr STATISTIK in ihren ersten sieben Kategorien stark mit der Einkommensverteilung, der Ausbildung und dem sozialen Prestige (BunDeSAMT Für Statistik 1995: 99f.). Dieses Merkmal ist daher als «erweiterter» Segregationsfaktor aufzufassen. Mit der Variablen «Haushalttyp» geht ebenfalls ein Indikator neuerer Gesellschaftstrends in die Untersuchung ein. Da das Konzept der gesellschaftlichen Milieus als soziale Mesoebene keine Differenzierung auf Personenebene verlangt, beziehen sich die Daten auf die Untersuchungseinheit «Haushalte»/ Angaben zum Haushaltsvorstand (RP) in Basel-Stadt. Sie entstammen dem Haushaltsregister der Schweizer Volkszählung von 1990 (99926 Haushalte; die Daten der schweizerischen Volkszählung 2000 sind zum Zeitpunkt der Abfassung des Artikels noch nicht verfügbar).

Durch Differenzierung der Segregationsanalysen nach verschiedenen räumlichen Bezugsebenen (Quartier und Baublock) wird der Massstab als Einflussgrösse auf Sozialraumprozesse berücksichtigt. So kann die in der Theorie postulierte Auflösung der Quartiergrenzen und die Unterteilung der Stadt in kleinere Einheiten segregierter Milieugruppen überprüft werden.

\subsection{Methodik}

Die Raumanalyse basiert auf mathematisch-statistischen Modellrechnungen der sozialräumlichen Segregation von Bevölkerungsgruppen mit einem Set von Indikatoren. Es gibt keine wissenschaftliche Einigung über Bestimmungsfaktoren und Messung der Segregation (Blasius 1988: 412f.). Die Berechnung des Segregationsindex erfolgt hier nach folgender Formel (verändert nach Friedrichs 1977: 220 und LichtenBERGER 1998: 242):

$S I=\frac{1}{2} \cdot \sum_{i=1}^{n} \cdot\left|\frac{a_{i}}{A}-\frac{m_{i}-a_{i}}{M-A}\right| \cdot 100$

Erläuterung;

$\mathrm{i}_{1}, \mathrm{i}_{2}, \ldots \mathrm{n}$ Zahl der Raumeinheiten (z.B. Baublock, Quartier)

$a_{i} \quad$ Bevölkerung der Gruppe a in der i-ten Raumeinheit

A Gesamtgrösse der Gruppe A im Gesamtgebiet

$\mathrm{m}_{\mathrm{i}} \quad$ Anzahl Bewohner in der i-ten Raumeinheit

M Anzahl Bewohner im Gesamtgebiet

Dieser Segregationsindex bestimmt die räumliche Ungleichverteilung einer Bevölkerungsgruppe im Vergleich zu allen anderen Gruppen derselben sozioökonomischen Kategorie bezogen auf eine bestimmte Raumeinheit. Je höher der Indexwert, desto grösser die relative Ungleichverteilung im Raum (SI $=0 \Rightarrow$ die betrachtete Gruppe ist gleich verteilt wie alle anderen
Gruppen derselben Kategorie; SI $=100 \Rightarrow$ die betrachtete Gruppe ist gegenüber allen anderen Gruppen maximal ungleich verteilt). Ein hoher Segregationsindex auf Quartier- und Baublockebene weist auf das Vorhandensein grösserer homogener Raumeinheiten hin. Bereits an dieser Stelle sei darauf hingewiesen, dass Raummassstab und Gruppengrösse den Indexwert verschieden stark verzerren können, z.B.:

- unterschiedliche Bevölkerungsanzahl der Teilgebietseinheiten: je weniger Haushalte in der Raumeinheit, desto grösser die Wahrscheinlichkeit der homogenen räumlichen Zusammensetzung oder

- unterschiedliche Grösse der Bevölkerungsteilgruppen: je kleiner die Gruppe im Vergleich zur Anzahl Raumeinheiten, desto grösser die Wahrscheinlichkeit, dass sie auf bestimmte Raumeinheiten konzentriert ist. Nähert sich die betrachtete Gruppe zahlenmässig der Anzahl Raumeinheiten oder unterschreitet sie diese gar, sind die Segregationswerte nicht mehr sinnvoll interpretierbar.

Bei der Analyse der Segregationswerte ist daher das Zahlenverhältnis Raumeinheit - Bevölkerungsteilgruppe zu beachten. Zudem ist die Homogenität von Baublöcken als Untersuchungseinheit nicht garantiert, da unterschiedliche Wohnsituationen von Strassenseiten oder Hinterhoflagen nicht berücksichtigt werden können.

Der Segregationsindex ist "nur» ein Mass für die Ungleichverteilung bezogen auf eine bestimmte Raumeinheit, er lässt keine Aussagen über die Lage der einzelnen sozialen Gruppen im Stadtgebiet zu. Für konkret raumbezogene Aussagen wird dieses räumlich abstrahierte Ergebnis durch eine Verortung der Segregationsmuster im Stadtraum ergänzt. Die kartographische Darstellung der anteilsmässigen Verteilung (quantile Klassierung) der untersuchten sozioökonomischen Variablen erfolgt durch das Geoinformationssystem ArcView ${ }^{\infty}$.

\subsection{Das Untersuchungsgebiet Basel}

Viertelstrukturen. Der Stadtkanton Basel besteht aus der Stadt Basel und den beiden Gemeinden Riehen und Bettingen (siehe Karte 1). Das Untersuchungsgebiet setzt sich aus sehr unterschiedlichen sozialen und räumlichen Viertelstrukturen zusammen. V.a. am nördlichen Stadtrand und im rechtsrheinischen Kleinbasel befinden sich dicht besiedelte Industrieund Arbeiterquartiere mit vielfach älteren kleineren Wohnungen und teilweise vernachlässigtem Unterhalt (Blockrandbebauung mit nachverdichteten Hinterhöfen). In diesen Quartieren mit hoher Immissionsbelastung aus Industrie und Verkehr sind z.T.Tendenzen zur Entstehung eines Prekärwohnungsmarktes vorhanden. Die Bevölkerung weist einen hohen Ausländeranteil (z.T. über $50 \%$ ) auf und setzt sich vornehmlich aus relativ jungen Bewohnern zusammen, die mittel bis weniger gut ausgebildet sind (Grossbasel: St. Johann, 


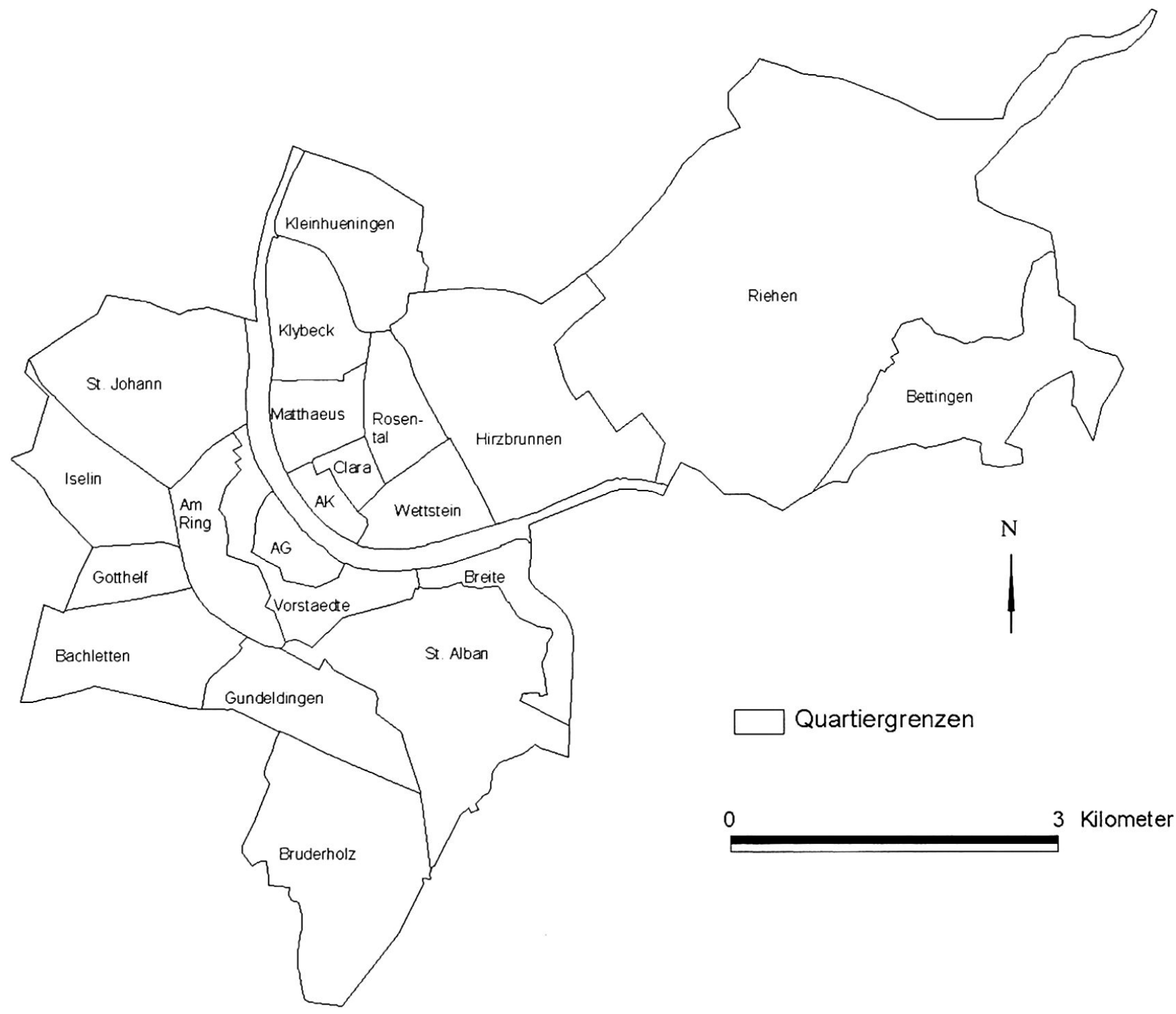

Karte 1: Quartiere und Landgemeinden des Kantons Basel-Stadt Urban quarters and rural communes of Canton of Basle-City Quartiers et communes rurales du Canton de Bâle-Ville Kartengrundlage: BundeSAMT FÜr STATISTIK GEOSTAT/L+T; Bearbeitung: S. EdER

Gundeldingen, Teile des Iselin; Kleinbasel: Matthäus, Klybeck, Kleinhüningen, Clara und Rosental).

Demgegenüber gibt es (luxus)sanierte Innenstadtquartiere («gentrifizierte» Altstadt Grossbasel, St. Alban) oder dünn besiedelte gründerzeitliche Villenviertel mit hohem Grünflächenanteil (Bachletten, Gellert/St. Alban) des gehobenen Mittelstands. Die Stadtrandgebiete mit Einfamilienhäusern und hohem Eigenheimanteil (Bruderholz: exklusive Wohnlagen am Hang mit grosszügigen Eigenheimen; Wettstein, Hirzbrunnen: Mittelstandwohngebiete mit Reihenhausbebauung) sowie die beiden Landgemeinden mit eher suburbaner Bebauungsstruktur und z.T. exklusiver Wohnlage am Hang sind bevorzugte Wohngebiete von (wohlhabenden) Familien und Rentnern. Die Viertel Am Ring, Breite, Vorstädte und Altstadt Kleinbasel weisen gemischte Wohnlagen und eine heterogen zusammengesetzte Sozialstruktur auf (SCHNEIDER-SLIWA ET AL. 1999: 52ff.).

Wirtschaftsstrukturen. Wirtschaftlich war Basel (Hauptindustriezweige Chemie und Bauwirtschaft) 1990 geprägt durch eine neue Konkurrenzsituation im Zuge der Globalisierung, verschärft durch rezessive Tendenzen der US-Wirtschaft und hohe Inflationsraten, einen Trend zur Tertiärisierung sowie weitreichende betriebswirtschaftliche Reorganisationen mit allgemei- 
nem Beschäftigtenabbau (dennoch lag die Erwerbslosenquote nur bei $1,2 \%$ ). Im Dienstleistungsgewerbe nahmen die Vollarbeitsplätze jedoch zu, v.a. in den Teilbereichen Versicherungen, Transport und Spedition, Beratung sowie Grossverteiler (FÜEG ET AL. 1992).

Bevölkerungsstrukturen. In Basel lassen sich die Trends der neuen Gesellschaftsentwicklung nachvollziehen. So hat sich die Stadtbevölkerung im Zuge der Counterurbanization seit den 70er Jahren um 16,4\% reduziert (SCHNEIDER-SLIWA ET AL. 1999: 8ff.). Seit Beginn der 90er Jahre wird eine Zunahme des Ausländeranteils beobachtet, der 1990 23,4\% betrug. 1990 überwogen die Nichtfamilienhaushalte mit 80,7\% gegenüber (Ehe-)Paaren mit Kindern mit 19,3\%. Der Anteil der Alleinerziehenden betrug 4,9\% (gleichbleibender Anteil seit 1980). In 70\% der Fälle ist die Situation der alleinerziehenden Eltern auf Scheidung zurückzuführen (SCHNEIDER-SLIWA ET AL. 1999: 27). Die Scheidungsrate lag 1990 insgesamt bei $10,6 \%$, $29,4 \%$ waren ledig. Nachweis über neue «entankerte», sich von Traditionen lossagende Lebensstilgruppen ist zudem der hohe Anteil an Konfessionslosen (1990 die stärkste «Konfessionsgruppe» mit 38\%; Protestanten: $32 \%$ ). Bei der sozio-professionellen Kategorie überwiegt der Anteil der Rentner mit $29 \%$. Den Trend zur Dienstleistungs- und Informationsgesellschaft belegen die nächststärkeren Gruppen der gelernten Arbeiter und Angestellten und der intermediären Berufe mit ihrem überwiegenden Anteil an Unternehmens- und Kommunikationsdienstleistungen. Die zunehmende Abwanderung einkommensstarker Bevölkerungsgruppen führt zu einer «A-StadtEntwicklung» im Kernstadtbereich (SCHNEIDER-SLIWA ET AL. 1999: 50).

\begin{tabular}{|l|c|c|c|c|c|}
\hline & \multicolumn{5}{|c|}{ Heimatstaat } \\
\hline & Schweiz & Mittel- und Nordeuropa & Südeuropa & $\begin{array}{c}\text { Südost- und Osteuropa, } \\
\text { Russland, Türkei }\end{array}$ & Sonstige Heimatländer \\
\hline $\begin{array}{l}\text { Gruppen- } \\
\text { stärke n }\end{array}$ & 79958 & 5237 & 8244 & 4896 & 1589 \\
\hline $\begin{array}{l}\text { SI Block- } \\
\text { ebene }\end{array}$ & 33,5 & 23,7 & 38,1 & 46,1 & 39,4 \\
\hline $\begin{array}{l}\text { SI Quar- } \\
\text { tierebene }\end{array}$ & 25,5 & 8,3 & 29,7 & 34,1 & 17,7 \\
\hline
\end{tabular}

\begin{tabular}{|l|c|c|c|c|c|c|c|c|c|}
\hline & \multicolumn{7}{|c|}{ Sozio-professionelle Kategorie } \\
\hline & $\begin{array}{c}\text { Oberstes } \\
\text { Mana- } \\
\text { gement }\end{array}$ & $\begin{array}{c}\text { Freie } \\
\text { Berufe }\end{array}$ & $\begin{array}{c}\text { Akademische } \\
\text { Berufe und } \\
\text { Obere Kader }\end{array}$ & $\begin{array}{c}\text { Andere } \\
\text { Selbständige }\end{array}$ & $\begin{array}{c}\text { Intermediäre } \\
\text { Berufe }\end{array}$ & $\begin{array}{c}\text { Qualifizierte } \\
\text { manuelle und } \\
\text { nicht-manuelle } \\
\text { Berufe }\end{array}$ & $\begin{array}{c}\text { Ungelernte } \\
\text { Arbeiter und } \\
\text { Angestellte }\end{array}$ & Erwerbslose & Rentner \\
\hline $\begin{array}{l}\text { Gruppen- } \\
\text { stärke n }\end{array}$ & 1059 & 959 & 7777 & 4815 & 18856 & 20215 & 10449 & 1222 & 29838 \\
\hline $\begin{array}{l}\text { SI Block- } \\
\text { ebene }\end{array}$ & & 33,4 & 25,6 & 15,4 & 16,5 & 35,4 & & 25,5 \\
\hline $\begin{array}{l}\text { SI Quar- } \\
\text { tierebene }\end{array}$ & 29,2 & 32,9 & 21,7 & 11 & 4,9 & 8,4 & 27,6 & 16,6 & 14,5 \\
\hline
\end{tabular}

\begin{tabular}{|l|c|c|c|c|c|c|c|}
\hline & \multicolumn{7}{|c|}{ Haushalttyp } \\
\hline Einzelpersonen & $\begin{array}{c}\text { Ehepaare } \\
\text { ohne Kinder }\end{array}$ & $\begin{array}{c}\text { Konsensualpaare } \\
\text { ohne Kinder }\end{array}$ & $\begin{array}{c}\text { Ehepaare mit 1 } \\
\text { oder 2 } \\
\text { Kind(ern) }\end{array}$ & $\begin{array}{c}\text { Ehepaare mit } \\
\text { 3 und mehr } \\
\text { Kindern }\end{array}$ & $\begin{array}{c}\text { Elternteile } \\
\text { mit Kind(ern) }\end{array}$ & $\begin{array}{c}\text { Nichtfamiliäre } \\
\text { Haushalte Nicht- } \\
\text { verwandter }\end{array}$ \\
\hline $\begin{array}{l}\text { Gruppen- } \\
\text { stärke n }\end{array}$ & 44018 & 22094 & 4228 & 15573 & 2820 & 4859 & 4598 \\
\hline $\begin{array}{l}\text { SI Block- } \\
\text { ebene }\end{array}$ & 21,4 & 20,7 & 24,3 & 21,7 & 39,5 & 22,4 & 29,1 \\
\hline $\begin{array}{l}\text { SI Quar- } \\
\text { tierebene }\end{array}$ & 11,4 & 13,2 & 7,4 & 10,3 & 15,9 & 6,1 & 16,4 \\
\hline
\end{tabular}

Tab. 1: Segregationsindices (SI) im Vergleich

Comparison of segregation indices (SI)

Indices comparés de ségrégation (SI)

Quelle: Statistisches Amt Kanton Basel-Stadt; Volkszählung 1990; eigene Berechnungen 


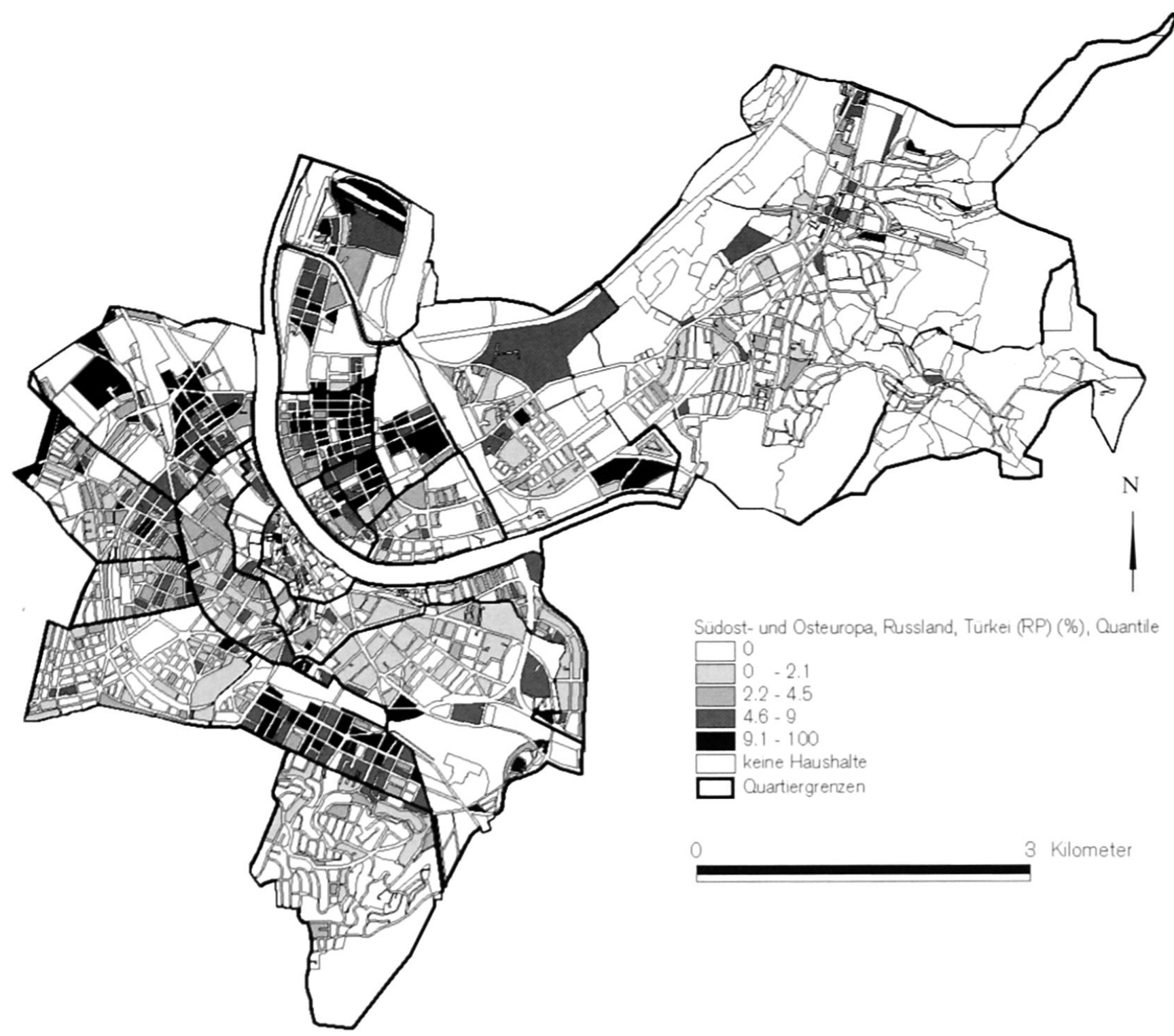

Karte 2: Residentielle Strukturmuster der Gruppe Südost- und Osteuropa. Russland. Türkei Residential structural patterns of the group "South-East and East Europe, Russia. Turkey" Modèles structurels résidentiels du groupe de l'Europe du Sud-est et de l'Est, de la Russie et de la Turquie Quelle: Statistisches Amt Kanton Basel-Stadt: Volkszählung 1990: Kartographie und Bearbeitung: S. Eder

\section{Ergebnisse und Diskussion}

Innerhalb der einzelnen sozioökonomischen Merkmalsgruppen Heimatstaat, sozio-professionelle Kategorie und Haushalttyp treten unterschiedlich hohe Indexwerte der residentiellen Segregation auf (siehe Tab. 1). Aufgrund des Einflusses von Grösse und Anzahl der Raumeinheiten sind auf der Quartierebene insgesamt geringere Indexwerte festzustellen als auf der Baublockebene. Bei diesem Massstab segregieren die kleineren Gruppen generell stärker als die grösseren. Werte für die Baublockebene werden nur für Gruppen dargestellt, deren Stärke die Anzahl Baublöcke $\mathrm{n}=1298$ übertrifft. Karten für die einzelnen Merkmale werden aus Platzgründen nur ausgewählt abgebildet.

\subsection{Heimatstaat}

Auf der Viertelebene ist die Gruppe "Südost- und Osteuropa. Russland, Türkei» am stärksten räumlich konzentriert. Sie segregiert auf beiden Massstabsebenen höher als die zahlenmässig stärker vertretenen Südeuropäer, die Mittel- und Nordeuropäer und die Schweizer. Migrationsmotiv dieser Bevölkerungsgruppe ist meist Asylsuche oder Familiennachzug. Sie wohnt va. in den dicht bebauten und emissionsbelasteten Arbeiter- und Industriequartieren der Stadt (siehe Karte 2). Die hohe Segregation nach Baublöcken weist zudem darauf hin. dass innerhalb dieser Quartiere nochmals eine Selektion nach Lage(un)gunst stattfindet: Auffallend viele Blöcke an verkehrsbelasteter Lage werden von dieser Gruppe bewohnt. Man kann davon ausgehen. dass der Wohnstandort hier weniger 


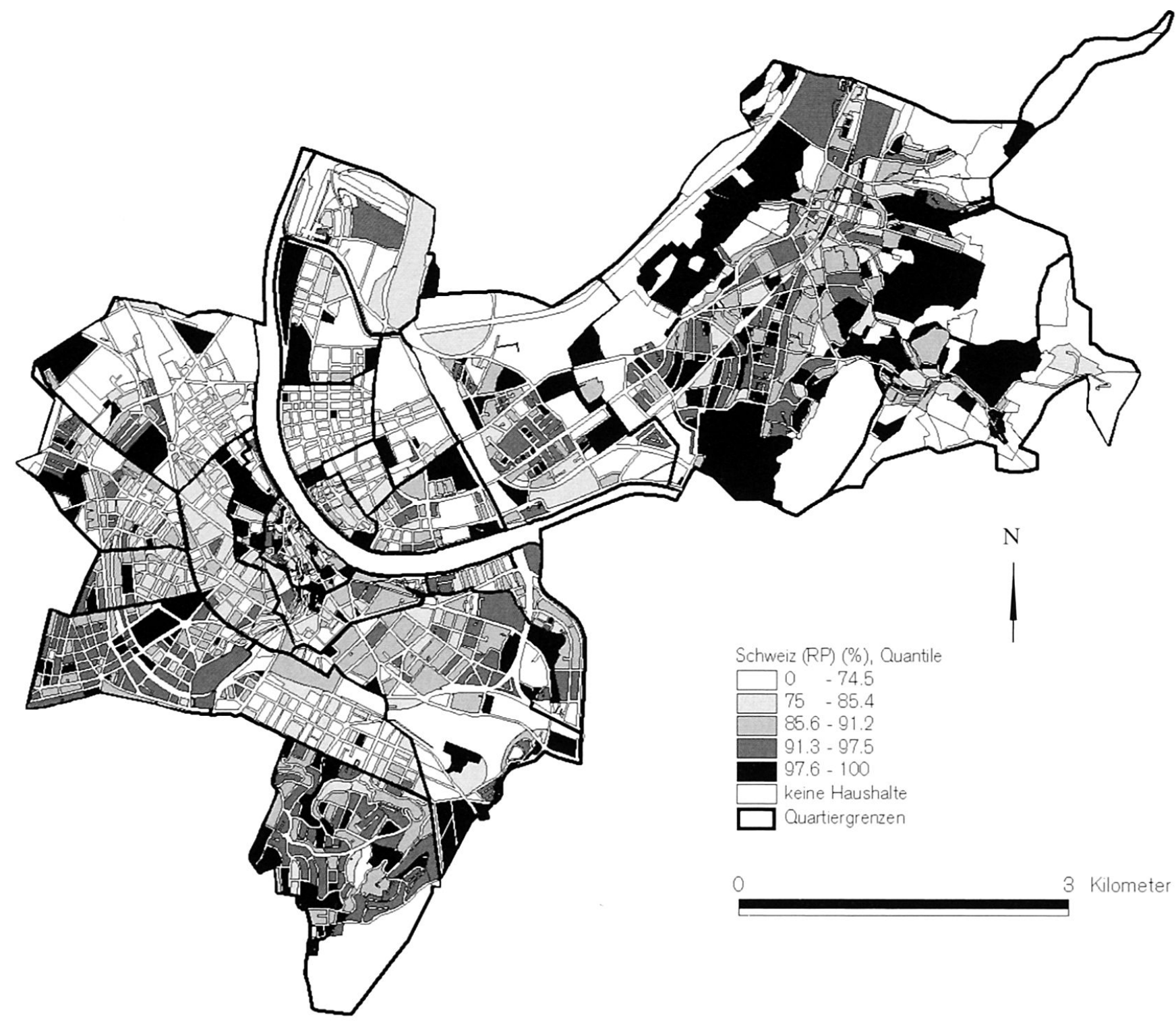

Karte 3: Residentielle Strukturmuster der Schweizer Residential structural patterns of the Swiss Modèles structurels résidentiels des Suisses Quelle: Statistisches Amt Kanton Basel-Stadt; Volkszählung 1990; Kartographie und Bearbeitung: S. Eder

durch Wahl als durch ökonomische Zwänge geprägt ist.

Die Südeuropäer, die als Arbeitsmigranten in die Stadt kamen, sind bei etwas geringeren Segregationswerten in sehr ähnlichem Muster über das Stadtgebiet verteilt. Korrelationsanalysen mit der sozio-professionellen Kategorie zeigen, dass beide Gruppen eher am unteren Ende der sozialen Leiter gesehen werden und damit auf das untere Segment des Wohnungsmarktes beschränkt sind. Die geringere räumliche Segregation der Südeuropäer verweist darauf, dass sie heute relativ frei in ihrer Wohnstandortwahl sind (vgl. ImHOF 1998: 88).

Bemerkenswert ist, dass die wesentlich grössere Gruppe der Schweizer auf beiden Massstabsebenen stärker segregiert wohnt als die Gruppe der Mittel- und
Nordeuropäer. Die Schweizer konzentrieren sich auf Gebiete mit qualitativ hochwertigem Wohnumfeld und hohem Sozialimage - aufgelockert bebaute städtische Randlagen mit hohem Eigenheimanteil oder repräsentative gründerzeitliche Villenviertel mit hohem Grünflächenanteil (siehe Karte 3). Kinderfreundlichkeit, Sicherheit, sowie die Lage zwischen Stadt und Land sind hier offensichtlich die Pullfaktoren. Schweizer Familien mit schulpflichtigen Kindern (Anteil: 14,2\%) ziehen aus dem rechtsrheinischen Kleinbasel, da sie Gewalt und ein vergleichsweise tiefes Lernniveau an den dortigen Schulen mit den üblicherweise gemischtnationalen Klassen befürchten. Der hohe Anteil an Schweizer Rentnern (35,4\%) zieht die ruhigen Stadtrandlagen als Wohnstandorte vor. Als Hauptpushfaktoren für den Wegzug der Schweizer aus Strassenzügen 


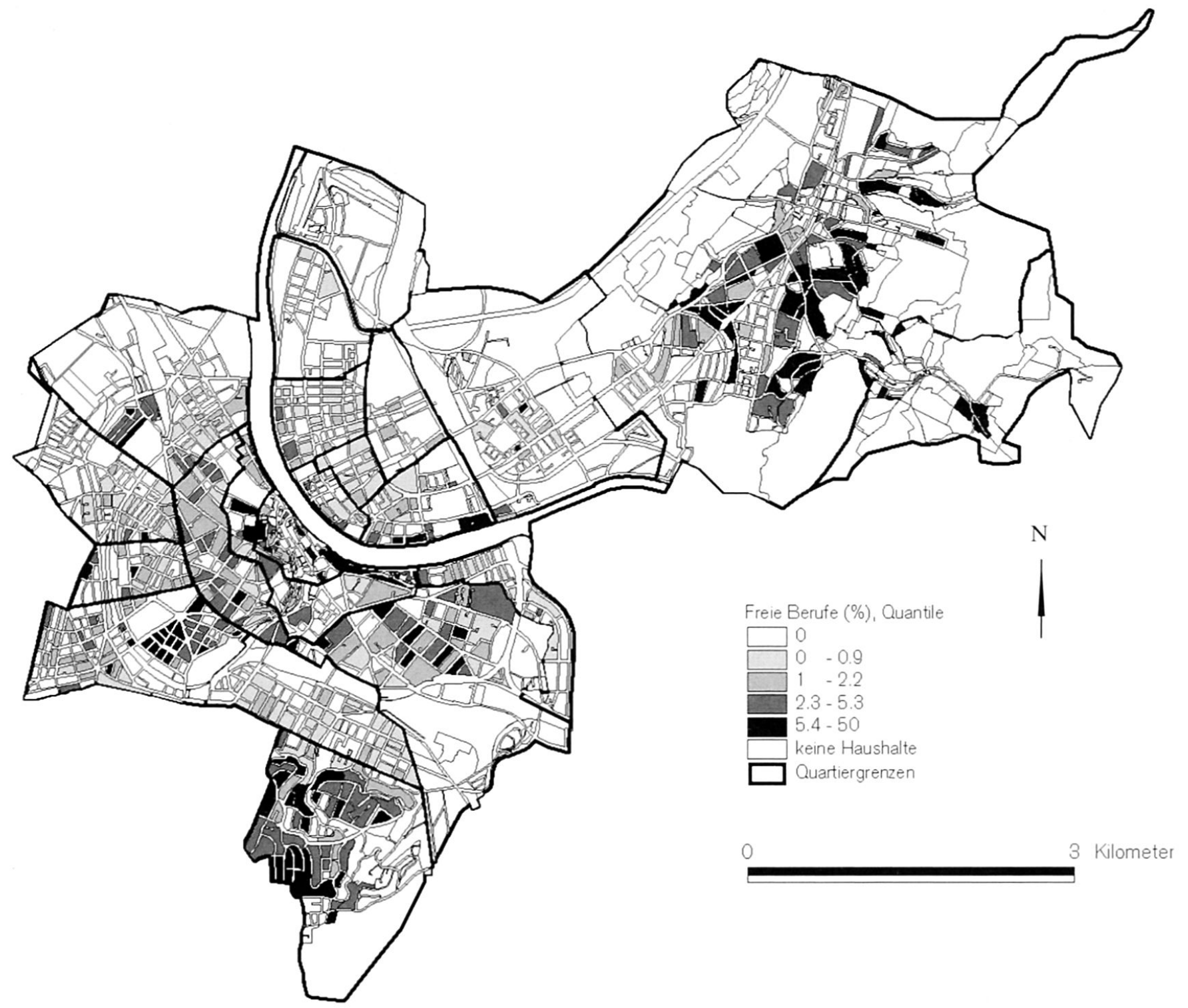

Karte 4: Residentielle Strukturmuster der Gruppe Freie Berufe

Residential structural patterns of the group "Independant Professions»

Modèles structurels résidentiels du groupe des professions libérales

Quelle: Statistisches Amt Kanton Basel-StadT; Volkszählung 1990; Kartographie und Bearbeitung: S. Eder

bestimmter Viertel nennt Iмноғ (Iмноғ 1998) die Vermeidung von Umweltbelastungen, v.a. Verkehrslärm. Die Mittel- und Nordeuropäer sind mit dem geringsten Segregationsindex auf Quartier- und Baublockebene relativ gleichmässig über die Stadt verteilt. Lediglich in sehr ungünstigen Wohnlagen ist ihr Anteil geringer. Korrelationsanalysen zeigen, dass diese Gruppe auch die oberen Segmente des Arbeitsmarktes besetzt und damit grösste Wahlfreiheit auf dem Wohnungsmarkt hat (oberstes Sozialprestige: $24,9 \%$ ). Als Ursache für das heterogene Raummuster könnte hier die heterogene sozioökonomische und -demographische Gruppenstruktur gesehen werden, wodurch sehr unterschiedliche Wohn(umfeld)ansprüche zum Tragen kommen. Allerdings weisen die Schweizer ebenfalls eine interne Heterogenität auf. Der Unterschied muss daher auf andere Faktoren, wie z.B. verschiedene Gruppenmentalität bezüglich des «intraethnischen Kontaktverhaltens», d.h. eine unterschiedliche Ausprägung des sozialräumlichen Zusammenlebens (MAHNIG 2001: 6), zurückgeführt werden. Die Segregationswerte der Sonstigen Heimatländer sind einerseits auf die geringe Gruppengrösse und andererseits auf die interne Heterogenität zurückzuführen. Auf eine weitere Interpretation der Ergebnisse wird daher verzichtet. 


\subsection{Sozio-professionelle Kategorie}

Die Untersuchung nach dem Merkmal der sozio-professionellen Kategorie führt zu dem Ergebnis, dass auf der Quartierebene die relativ kleinen Gruppen der statushöchsten Berufsfelder der Freien Berufe (siehe Karte 4), gefolgt vom Obersten Management, am stärksten segregieren. Diese einkommensstarken Gruppen sind räumlich auf die exklusivsten Stadtquartiere mit dem besten Viertelimage konzentriert (städtische Randlagen, luxussanierte Innenstadt- und Altstadtlagen). Sie können sich eine Prestigedemonstration durch selektive Wohnstandortwahl leisten. Die statushohe Gruppe der Akademiker und Oberen Kader segregiert auf beiden Massstabsebenen weniger intensiv, aber immer noch vergleichsweise hoch. Ihre Verteilung deckt sich räumlich mit den beiden erstgenannten Gruppen, wobei sie selektiv Baublöcke mit bevorzugter Wohnqualität (grosszügiger Wohnraum, hoher Eigenheimanteil und Gärten) in Quartieren mit gemischten Wohnlagen bewohnt.

Der dritthöchste Segregationsindex wird für die grosse Gruppe der ungelernten Arbeiter und Angestellten erreicht. Sie konzentriert sich stark auf die Arbeiter- und Industriequartiere mit niedriger Wohnumfeldqualität. Hohe Segregationsindexwerte auf Baublockebene deuten darauf hin, dass dieser Segregationsprozess zudem durch preisgünstige Wohnungen an qualitativ geringwertigen Lagen innerhalb

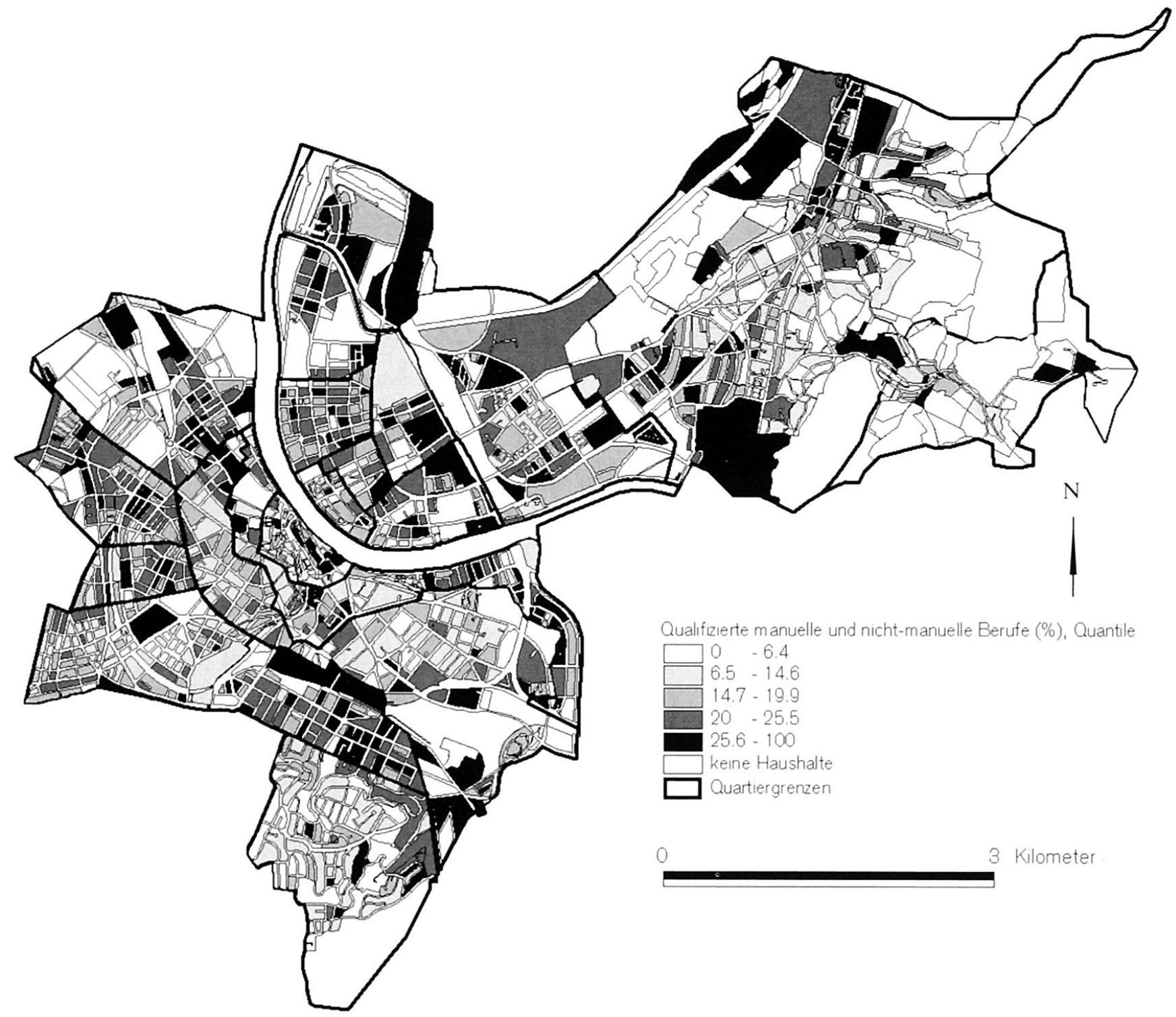

Karte 5: Residentielle Strukturmusert der Gruppe Qualifizierte manuelle und nicht-manuelle Berufe Residential structural patterns of the group "Qualified manual and non-manual professions" Modèles structurels résidentiels du groupe des professions qualifiées manuelles et non-manuelles Quelle: Statistisches Amt Kanton Basel-Stadt; Volkszählung 1990; Kartographie und Bearbeitung: S. Eder 
der Viertel, z.B. an verkehrsbelasteten Strassenzügen oder schlecht unterhaltenen Wohnobjekten, beeinflusst ist.

Die Erwerbslosen können sich keine Wohnstandortwahl leisten, sie konzentrieren sich räumlich auf einzelne Baublöcke in Arbeiter- und Industrievierteln sowie ungünstige Wohnlagen innerhalb anderer Stadtquartiere.

Der Segregationsindex der Rentner stellt sich vergleichsweise niedrig dar. Hier fällt die Gruppengrösse stark ins Gewicht. Ein Indiz für die dennoch starke Segregation dieser Bevölkerungsgruppe ist, dass sie einen höheren Segregationsindex aufweist als die kleinere Gruppe der Qualifizierten manuellen und nichtmanuellen Berufe. Die Rentner weisen ein deutlich nach Vierteln segregiertes residentielles Muster auf, ihre Wohnlagen sind auf die im Vergleich zur Innenstadt ruhigen Stadtrandlagen mit höherem Sicherheitsimage verteilt.

Von den «mittleren» Berufskategorien segregiert die relativ kleine Gruppe der Anderen Selbständigen deutlich stärker als die zahlenmässig weit überlegenen Intermediären Berufe und die Qualifizierten manuellen und nicht-manuellen Berufe (siehe Karte 5).

Die vergleichsweise niedrigen Segregationsindices auf der Viertelebene weisen darauf hin, dass diese Gruppen weniger durch das Quartierimage beeinflusst sind. Das residentielle Strukturmuster ist dementsprechend eher stadtweit kleinräumig-clusterartig. Mit abnehmendem Sozialprestige und Einkommen von den

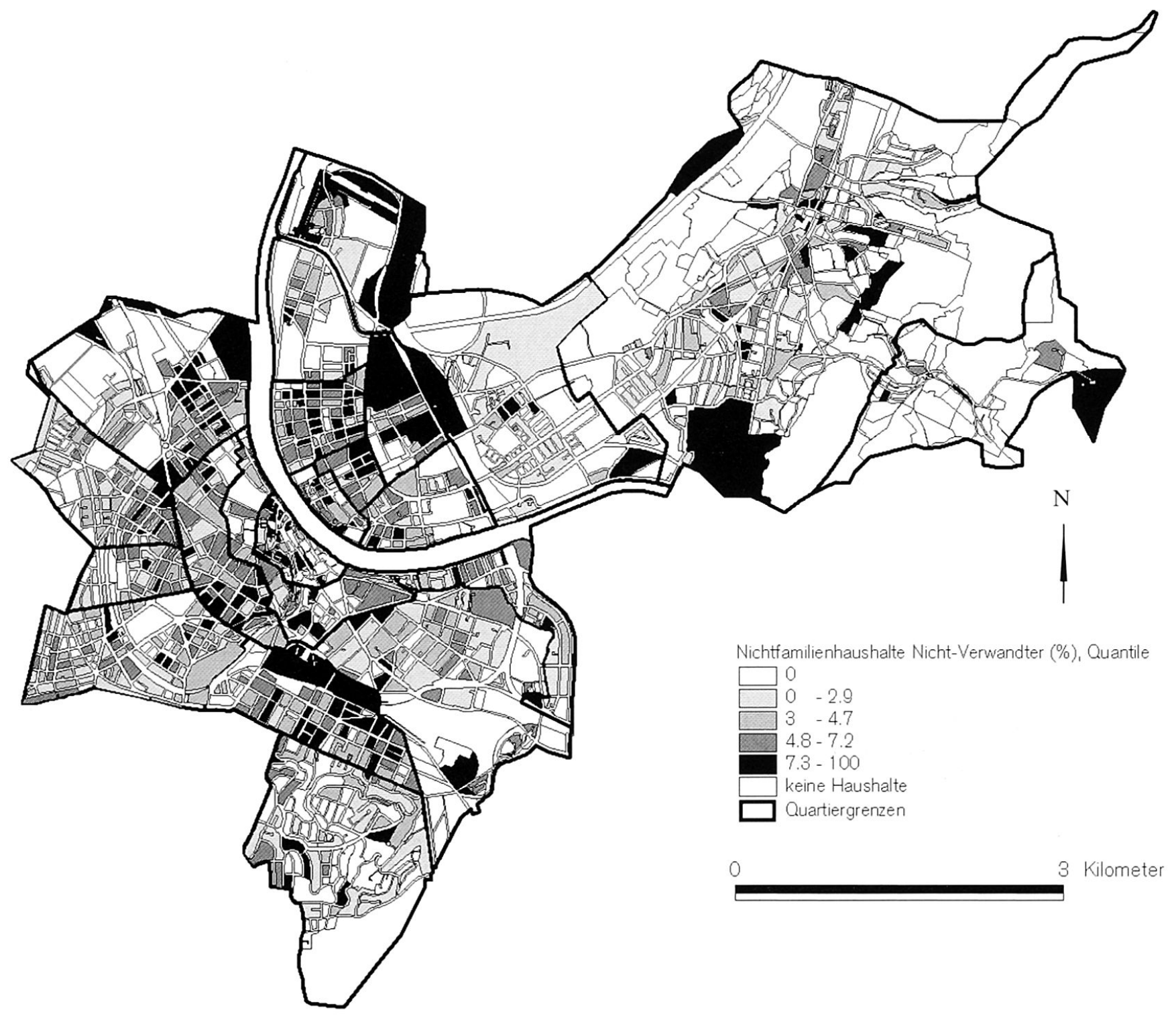

Karte 6: Residentielle Strukturmuster der Gruppe Nichtfamilienhaushalte Nicht-Verwandter

Residential structural patterns of the group «Non-family/Not related households»

Modèles structuresl résidentiels du groupe des ménages non-familiaux et non-apparentés

Quelle: Statistisches Amt Kanton Basel-Stadt; Volkszählung 1990; Kartographie und Bearbeitung: S. Eder 
Anderen Selbständigen über die Intermediären Berufe zu den Qualifizierten manuellen und nicht-manuellen Berufen verlagern sich die Verteilungsschwerpunkte immer mehr von den privilegierteren Quartieren hin zu den Arbeiter- und Industriequartieren. Der deutlich höhere Segregationswert der Anderen Selbständigen auf Blockebene verweist auf eine grössere Freiheit dieser Gruppe bei der Wohnstandortwahl, während die leicht höheren Werte der Qualifizierten manuellen und nicht manuellen Berufe gegenüber den Intermediären Berufen auf beiden Massstabsebenen auf eine stärkere Einschränkung der Wahlfreiheit hindeuten.

\subsection{Haushalttypen}

Den höchsten Segregationsindexwert auf der Quartierebene haben für diese Variablenkategorie die Nicht-Familienhaushalte Nicht-Verwandter (Wohngemeinschaften; siehe Karte 6). Die verhältnismässig junge Gruppe (52\% sind zwischen 15 und 30 Jahre alt) verteilt sich v.a. auf innenstadtnahe Lagen und die Altstadt. Bevorzugte Wohnviertel sind v.a. Arbeiter- und Industriequartiere. Entscheidend für die Wohnstandortwahl dieser sehr mobilen, da nicht durch Familie gebundene Haushaltsform, sind günstige Quadratmetermieten kombiniert mit zentrumsnahen Lagen, guter Versorgungssituation und urbaner Ausstrahlung.

Nächsthöherer Segregationsindex auf der Quartierebene wird von Familien mit drei und mehr Kindern erreicht. Diese relativ kleine Gruppe ist in ihrer Wohnortwahl durch ihren hohen Flächenanspruch in kinderfreundlicher Umgebung v.a. auf städtische Randlagen eingeschränkt. Die sehr starke Segregation auf Baublockebene ist darauf zurückzuführen, dass innerhalb der Stadtviertel die Wohnansprüche nur in einzelnen Blöcken befriedigt werden können.

Familien mit einem oder zwei Kindern segregieren weniger stark als die zahlenmässig stärkeren Gruppen der Ehepaare ohne Kinder («DINKS» - double income no kids) und der Einpersonenhaushalte. Die letztgenannten Gruppen sind ökonomisch gut gestellt und können die Wohnsituation ihren jeweiligen Bedürfnissen entsprechend frei wählen. Die Einpersonenhaushalte weisen ein fast identisches Verteilungsmuster wie die Nicht-Familienhaushalte Nicht-Verwandter auf (innenstadtnahe Gunstlagen), was auf einen ähnlichen Lebensstil zurückzuführen ist. Ehepaare ohne Kinder sind wie die Familien mit 1 oder 2 Kindern eher in gehobenen Stadtrandlagen mit hohem Grünflächenanteil und lichter Bebauungsstruktur zu finden. Auf Baublockebene segregieren die Familien leicht stärker, was auf deren spezifischeren Wohnraumbedarf schliessen lässt.

Erstaunlicherweise segregieren die zahlenmässig schwächeren und finanziell sehr gut gestellten Konsensualpaare ohne Kinder («DINKS») weniger stark. Sie sind relativ gleichmässig über das gesamte Stadtgebiet mit Ausnahme der beiden Landgemeinden und der weniger guten Lagen in den Industrieund Arbeiterquartieren verteilt. Ihr vergleichsweise hoher Segregationsindex auf Baublockebene und das eher clusterartige Verteilungsmuster verweisen darauf, dass für diese «sozial entankerte» Gruppe der Quartiercharakter eine eher unbedeutende Rolle spielt, wohl aber die direkte Wohnlage innerhalb der Viertel.

Am schwächsten segregieren die Alleinerziehenden auf der Viertelebene. Sie sind in innenstadtnahen Lagen ebenso anzutreffen wie in Stadtrandquartieren. Erklärung hierfür könnte die ökonomische Heterogenität dieser Gruppe sein. Während die einen am ehemaligen Familienwohnsitz verbleiben können, sind die anderen gezwungen, in preisgünstigere Wohnungen umzuziehen.

\section{Zusammenfassende Betrachtungen}

In dieser Studie kann keine generelle Auflösung der «Lehrbuchstrukturen» des städtischen Sozialraums (s.o.) festgestellt werden, da die einzelnen Sozialindikatoren unterschiedlich segregieren. Jede der verwendeten Variablenkategorien beinhaltet Gruppen, die eher homogene und damit grossräumige residentielle Raummuster bilden und Gruppen, die eher kleinräumig heterogene Wohnortstrukturen aufweisen. Bei einem Vergleich der Indices der einzelnen Teilgruppen ist jedoch stets zu beachten, dass deren unterschiedliche Grösse einen beachtlichen Einfluss auf das Rechenergebnis hat (s.o.). Womit diese Unterschiede im einzelnen zusammenhängen, wird im folgenden für die einzelnen Variablen dargestellt.

\section{Heimatstaat:}

- Hier ist die Gruppengrösse nicht ausschlaggebend für das Segregationsausmass.

- Entscheidend ist eine unterschiedliche Gruppenmentalität bezüglich des sozialen Kontakt- bzw. Wohnverhaltens.

- Zudem schränkt der unterschiedliche ökonomische Status der Nationalitäten die Wohnstandortwahl auf bestimmte Stadtgebiete ein oder lässt Wahlfreiheiten zu.

- Generell weisen soziodemographisch und -ökonomisch homogenere Ethniegruppen höhere Segregationswerte auf als heterogene Teilgruppen.

\section{Sozio-professionelle Kategorie:}

- Hier zeigt sich, dass die räumliche Ungleichverteilung der Wohnbevölkerung stark vom Einkommensniveau bzw. dem sozialen Prestige abhängt: Grösste Wahlfreiheit auf der einen Seite und ökonomische Zwänge bis Zugangsbarrieren auf Teilwohnungsmärkten auf der anderen Seite führen zu grösseren homogenen Raumeinheiten.

- Der Wohnort der sozioökonomisch unterprivilegierten oder marginalisierten Gruppen sowie bestimm- 
ter Teile der Privilegierten wird durch die gegebene Raumausstattung sowie (für die Bessergestellten) das Image der Viertel bestimmt; Raumeinflüsse überwiegen hier die rein durch gesellschaftliche Faktoren beeinflusste sozialräumliche Entmischung.

- Berufsgruppen mit mittlerem Sozialstatus sind auf der Viertelebene geringer segregiert, entscheidend für die Wohnstandortwahl ist eher die direkte Wohnumgebung des Baublocks, ihre Standortmuster sind dementsprechend heterogen-clusterartig im Stadtraum verteilt.

\section{Haushalttyp:}

- Der Trend zur Auflösung der sozialräumlichen Homogenität zonaler und sektorieller Raummuster und zur kleinräumig-inselhaften Heterogenität ist hier nur für bestimmte «modernisierte» bzw. «sozial entankerte» Merkmalsgruppen feststellbar.

- Die ebenfalls als «moderne Lebensformen» einzustufenden Einpersonenhaushalte und Nichtfamilienhaushalte Nicht-Verwandter segregieren dagegen mit ihrer Bevorzugung bestimmter Stadtviertel relativ hoch.

- Eher «traditionell» ausgerichtete Merkmalsgruppen sind ebenfalls vorwiegend in herkömmlich grossflächigeren Segregationsmustern im Stadtraum verteilt (Familien, Ehepaare ohne Kinder).

- Die Heterogenisierung der städtischen Sozialraummuster hängt also nur bedingt mit der gesellschaftlichen «Modernisierung» zusammen (zutreffend für Konsensualpaare). Entscheidender ist die interne Ähnlichkeit einer Gruppe bezüglich ihrer Wohnortansprüche: Die Wohnstandortwahl beschränkt sich dann auf bestimmte Raumeinheiten (Familien, Nichtfamilienhaushalte Nicht-Verwandter, Einpersonenhaushalte, Rentner). Intern uneinheitliche Gruppen finden dagegen an verschiedenen Wohnstandorten in unterschiedlichen Vierteln eine geeignete Wohnumgebung (Alleinerziehende).

\section{Soziale Lage:}

- Die soziale Lage hat immer noch einen wesentlichen Einfluss auf die sozialräumliche Segregation.

- Zudem wirken sich demographische Eigenschaften und interne Zusammensetzung der jeweiligen Merkmalsgruppe auf den Segregationsprozess aus.

- Heterogen-clusterartige Raummuster infolge selektiver Ansprüche an die direkte Wohnumgebung sind v.a. bei Bevölkerungsgruppen anzutreffen, die aufgrund ihrer finanziellen und demographischen Voraussetzungen flexibel auf das städtische Wohnraumangebot reagieren (können oder wollen): v.a. Gruppen mit mittlerem Sozialprestige und Einkommen, deren Wohnstandortwahl nicht so stark vom Viertelcharakter, sondern von individuellen Vorlieben und Möglichkeiten geprägt ist. Von diesen Gruppen werden städtische Teilräume neu bewertet und «sozial umgenutzt».

- Sozioökonomisch privilegierte und benachteiligte
Gruppen segregieren dagegen aufgrund ihrer erhöhten Wahlfreiheit bzw. deren Einschränkung stärker nach Quartieren.

- Der Aspekt der gesellschaftlichen «Modernität» spielt also nur für bestimmte wohlhabendere Gruppen eine nachgeordnete Rolle bei der residentiellen Segregation. Sozioökonomische und -demographische Heterogenität (s.o.) wirkt sich dagegen stark auf den Prozess der sozialräumlichen Heterogenisierung in ein kleinräumiges «Mosaik aus sozialen Inseln» aus.

Die physisch-materielle Raumstruktur hat einen unterschiedlich dominanten Einfluss auf die sozialräumliche Verteilung der Stadtbevölkerung:

- Für Gruppen, die sozialräumlich in relativ homogene Stadtviertel segregieren, sind die physischmateriellen Raumgegebenheiten des gesamten Quartiers entscheidend für die Wohnstandortwahl.

- Gruppen, die sich sozialräumlich heterogen im Stadtgebiet verteilen, lassen sich in ihrer Wohnstandortwahl dagegen eher von der konkreten Wohnsituation als vom Quartiercharakter leiten.

- In Basel sind gesellschaftliche Segregationserscheinungen - seien sie bewusst gewählt oder unfreiwillig durch sozioökonomische Zwänge verursacht nicht nur in strukturell benachteiligten, sondern auch in strukturell bevorzugten Stadtteilen zu finden. Es ist ein Ergebnis dieser Studie, dass sowohl einseitig negative wie auch positive Viertelstrukturen eine residentielle Segregation fördern können.

\section{Ghettoisierung:}

Eine «Ghettoisierung» kann für Basel nicht festgestellt werden, auch wenn sich Hinweise auf räumliche Konzentration unterprivilegierter Gruppen in benachteiligten Wohnvierteln ergeben (Kleinbasel, St. Johann). Der Begriff «Ghetto» stammt aus der nordamerikanischen Stadtforschung und ist aus verschiedenen Gründen für westeuropäische Städte verfehlt (MAHNIG 2001:2):

- Die sog. «Migrantenviertel» in Basel sind vergleichsweise kleinräumig und nicht nur von einer einzigen Immigrantengruppe bewohnt, der Anteil an einheimischer Bevölkerung bleibt jeweils hoch.

- Da diese Quartiere nicht zugleich Arbeits- und Wohnort sind, sind die Bewohner in ihren Aktionsradien nicht auf diese Stadtsegmente beschränkt, so dass es nicht zu sozialer Isolation kommt.

\section{Methodische Weiterentwicklung zur Erfassung von Segregationserscheinungen}

Methodisch muss an der Berechnung der residentiellen Entmischungserscheinungen durch die verwendete Segregationsformel bemängelt werden, dass sie die Grössenunterschiede der Teilgruppen zu stark gewichtet (ausführlich erläutert in Kap. 3.2). Dies kann zu 
einer verfälschenden Aussage der Segregationsindices verleiten: Die hohen Segregationsindexwerte auf der Baublockebene für sehr kleine Bevölkerungsgruppen beruhen auf deren eher zufälligen Verteilung im Stadtraum, sie sind nicht mit den hohen Indices grosser Gruppen zu vergleichen.

Da die Formel den Raumbezug ausser Acht lässt, muss zur fundierten Stadtraumanalyse eine kartographische Verortung auf der kleinsten untersuchten Raumeinheit ergänzt werden. Umgekehrt sind auch Karten der Bevölkerungsverteilung allein oft schwer zu interpretieren, v.a. wenn sich die Raummuster (z.B. bei kleinen Gruppen) nicht klar an Viertelgrenzen orientieren. Der komplementäre Einsatz der beiden Methoden erscheint daher sinnvoll.

\section{Ausblick}

Als Fazit der vorliegenden Segregationsstudie kann festgehalten werden, dass die residentielle Segregation in Basel von sozialen Bedingungen wie Ethnie, sozialer Lage und Haushalttyp abhängt. Dabei segregieren die Untergruppen dieser Merkmalskategorien verschieden stark und führen zu unterschiedlichen Raummustern, je nach «Modernisierungsgrad», interner Homogenität und sozioökonomischem Status.

Gleichzeitig wird der Prozess geleitet durch eine bestimmte - stadtentwicklungsgeschichtlich vorgegebene - Raumkonstellation unterschiedlichen Wohnraumangebots. Dieses Ergebnis unterstützt die heute von Soziologen vertretene Überzeugung, dass dem Trend der sozialräumlichen Segregation eher mit Programmen gegen die sozioökonomische Benachteiligung, v.a. gegen die Diskriminierung auf dem Wohnungs- und Arbeitsmarkt, als mit Durchmischungspolitik begegnet werden sollte (vgl. MaHNIG 2001: 1). Für die stadtpolitisch gewünschte sozialräumliche Integration ist es in diesem Zusammenhang auch wichtig, kleinräumige Wohnlageunterschiede bzw. Vielfalt im Wohnungsmarkt zu schaffen und zu erhalten. Dies kann z.B. durch die Produktion von grosszügigem modernen Wohnraumangebot an günstiger Lage (abseits von Verkehrs- und Umweltbelastung) in gemischtstrukturierten Wohnvierteln oder in Quartieren mit traditionell negativem Image geschehen, so dass soziodemographisch heterogene Bevölkerungsgruppen der mittleren und oberen sozialen Lagen stadtweit geeigneten Wohnraum, unabhängig vom Viertelgesamtcharakter oder -image, finden können.

\section{Literatur}

BACKHAUS, N. (1999): Zugänge zur Globalisierung - Konzepte, Prozesse, Visionen. - = Schriftenreihe Anthropogeographie 17, Zürich: Druckerei der Zentralstelle der Studentenschaft der Universität Zürich. Blasius, J. (1988): Indices der Segregation. - In: FiRED-
RICHS, J. (Hrsg.): Soziologische Stadtforschung. - Opladen: 410-431.

Borchers, S. \& G. Tempel (1998): Freizeitstile in einer Grossstadt - eine empirische Untersuchung. - = Arbeitspapiere 31, Bremen: Universität Bremen.

Bourdieu, P. (1983): Ökonomisches Kapital, kulturelles Kapital, soziales Kapital. - In: KrecKel, R. (Hrsg.): Soziale Ungleichheiten. - Göttingen: 183-198.

Bourdieu, P. (1991): Physischer, sozialer und angeeigneter Raum. - In: WenTZ, M. (Hrsg.): Stadträume. Frankfurt a.M.: 25-34.

Bundesamt Für StatistiK (Hrsg.) (1995): Eidgenössische Volkszählung 1990. Sozialstruktur der Schweiz. Sozio-professionelle Kategorien. - = Statistik der Schweiz, Bern: Bundesamt für Statistik.

Castells, M. (1994): European cities, the informational society, and the global economy. - In: New Left Review 204: 18-32.

Dangschat,J.S. (1994): Multikulturelle Stadt und soziale Polarisierung. - In: Schwartz, U. (Hrsg.): Risiko Stadt? Perspektiven der Urbanität. - Hamburg: 177-190.

Dangschat, J. S. (1998): Klassenstrukturen im NachFordismus. - In: Berger, P. A. \& M. Vester (Hrsg.): Alte Ungleichheiten neue Spaltungen. - = Sozialstrukturanalyse 11, Opladen: 49-87.

Friedrich, M. (1999): Die räumliche Dimension städtischer Armut. - In: DangSchat, J.S. (Hrsg.): Modernisierte Stadt - gespaltene Gesellschaft. Ursachen von Armut und sozialer Ausgrenzung. - Opladen: 263-287.

Friedrichs, J. (1977): Stadtanalyse. Soziale und räumliche Organisation der Gesellschaft. - Reinbeck bei Hamburg: Rowohlt.

FÜEg, R. ET AL. (1992): Regio Wirtschaftsstudie Nordwestschweiz XIII. - = Schriften der Regio 7.13, Basel: Helbing und Lichtenhahn.

HahN, G. M. (1999): Sozialstruktur und Armut in der nach-fordistischen Gesellschaft. Ökonomische Polarisierung und kulturelle Pluralisierung als Aspekte struktureller Marginalisierungsprozesse. - In: DANGSCHAT, J.S. (Hrsg.): Modernisierte Stadt - gespaltene Gesellschaft. Ursachen von Armut und sozialer Ausgrenzung. - Opladen: 179-212.

Helbrecht, I. (1997): Stadt und Lebensstil. Von der Sozialraumanalyse zur Kulturraumanalyse? - In: Die Erde 128: 3-16.

Herlyn, U. \& A. Harth (1996): Soziale Differenzierung und soziale Segregation. - In: StRubelt, W. ET AL. (Hrsg.): Städte und Regionen. - = Berichte zum sozialen und politischen Wandel in Ostdeutschland, Opladen: 257-287.

Herlyn, U., Lakemann, U. \& B. Lettko (1991): Armut und Milieu: benachteiligte Bewohner in grossstädtischen Quartieren. - = Stadtforschung aktuell 33, Basel, Boston, Berlin: 259.

HRADIL, S. (1987): Sozialstrukturanalyse einer fortgeschrittenen Gesellschaft. Von Klassen und Schichten 
zu Lagen und Milieus. - Opladen: Leske und Budrich. HradiL, S. (1990): Von der «Nivellierten Mittelstandsgesellschaft» zur «Pluraldifferenzierten Wohlstandsgesellschaft». - In: ANDERSEN, U., GAGEL, W. \& P. HAUnGS (Hrsg.): Soziale Differenzierung - soziale Ungleichheit. $-=$ Politische Bildung 23,2, Stuttgart: 18-50.

Hradil, S. (1999): Soziale Ungleichheit in Deutschland. - Opladen: Leske und Budrich.

IмноF, M. (1998): Migration und Stadtentwicklung. Aktualgeographische Untersuchungen in den Basler Quartieren Iselin und Matthäus. - = Basler Beiträge zur Geographie 45, Dissertation, Geographisches Institut, Universität Basel, Basel.

KRÄTKE, S. (1991): Strukturwandel der Städte: Städtesystem und Grundstücksmarkt in der "post-fordistischen» Ära. - Frankfurt a.M.: Campus Verlag.

KRÄTKE, S. (1997): Globalisierung und Stadtentwicklung in Europa. - In: Geographische Zeitschrift, 85. Jahrgang, Heft 2/3: 143-158.

LeFEBVRE, H. (1991):The production of space.- Oxford: Blackwell.

LiCHTENBERGER, E. (1998): Stadtgeographie. - = Teubner Studienbücher Geographie 1, Stuttgart: Teubner. MAHNig, H. (2001): Ethnische Segregation als Herausforderung städtischer Politik. Thesenpapier. - Schweizerisches Forum für Migrationsstudien, Neuchâtel.

Marcuse, P. (1995): Not Chaos, but Walls: Postmodernism and the Partitioned City. - In: Watson, S. \& K. Gibson (Hrsg.): Postmodern Cities and Spaces. - Cambridge, Ma.: 243-253.

MüLLER, H.-P. (1989): Lebensstile. - In: Kölner Zeitschrift für Soziologie und Sozialpsychologie 41: 53-71. MülLER, H.-P. (1992): Sozialstruktur und Lebensstile. - In: Hradil, S. (Hrsg.): Zwischen Bewusstsein und Sein. Die Vermittlung «objektiver» und «subjektiver» Lebensweisen. - Opladen: 57-66.

Noller, P. (1999): Globalisierung, Stadträume und Lebensstile. Kulturelle und lokale Repräsentation des globalen Raums. - Opladen: Leske und Budrich.

SCHELSKI, H. (1965): Auf der Suche nach Wirklichkeit. - Düsseldorf, Köln: Eugen Diederichs.

SChNeider-Sliwa, R. ET AL. (1999): Bevölkerungsstruktur und Bevölkerungsdynamik beider Basel. - = Stadt und Region 1, Basel: Statistisches Amt des Kantons Basel-Stadt.

SoJA, E. (1995): Postmodern Urbanisation: the Six Restructurings of Los Angeles. - In: Watson, S. \& K. Gibson (Hrsg.): Postmodern Cities and Spaces. - Cambridge, Ma.: 125-137.

SPELLERBERG, A. (1997): Lebensstile und Wohnverhältnisse FS III 97 - 403. - Wissenschaftszentrum Berlin für Sozialforschung, Abteilung Sozialstruktur und Sozialberichterstattung, Berlin.

Vester, M. (1993): Soziale Milieus im gesellschaftlichen Strukturwandel. Zwischen Integration und Ausgrenzung. - Köln: Bund-Verlag.

Vester, M. (1998): Klassengesellschaft ohne Klassen.
Auflösung oder Transformation der industriegesellschaftlichen Sozialstruktur? - In: BERGER, P.A. \& M. Vester (Hrsg.): Alte Ungleichheiten neue Spaltungen. - = Sozialstrukturanalyse 11, Opladen: 109-147.

WERLEN, B. (1995): Sozialgeographie alltäglicher Regionalisierungen. Zur Ontologie von Gesellschaft und Raum. Band 1. - = Erdkundliches Wissen 116, Stuttgart: 1- 262.

WYLY, E.K. (1999): Continuity and Change in the Restless Urban Landscape. - In: Economic Geography 75, Heft 4: 309-338.

\section{Zusammenfassung: Städtische Sozialstrukturen und residentielle Segregationsmuster am Beispiel Basel- Stadt}

Neuere gesamtgesellschaftliche Strukturveränderungen haben zu einem veränderten Sozialratiiı fefüge der Stadt geführt. Um den theoretisch postulierten Prozess der sozialräumlichen Heterogenisierung in ein kleinräumiges Mosaik sozialer Milieus zu dokumentieren, wird die sozialräumliche Segregation verschiedener Bevölkerungsgruppen in Basel-Stadt empirisch erfasst und analysiert. Es zeigt sich, dass gesellschaftliche «Modernität», die soziale Lage sowie interne Heterogenität der betrachteten Gruppen einen entscheidenden Einfluss auf die Entstehung der neuen Raummuster haben. Aspekte der Wohnumfeldqualität geben Aufschluss über den Einfluss des Raums auf die sozialen Abgrenzungs- bzw. Konzentrationsprozesse.

\section{Summary: Urban Social Structures and Patterns of Residential Segregation in Basle, Switzerland}

Recent social transformations have led to a changing structure in urban social space. Social scientists postulate the dissolution of formerly homogenous city quarters into a manifold spatial mosaic of different lifestyle groups. This study empirically documents and analyses social segregation processes for different social groups in the city of Basle. Social modernity, social standing and internal heterogeneity of the respective social group turn out to have an important influence on the creation of «new» spatial structures. The residential surroundings are included in the investigation in order to assess the impact of space on processes of social segregation.

\section{Résumé: Structures sociales urbaines et modèles de ségrégation résidentielle: le cas de Bâle-Ville}

De récentes transformations structurelles sociétales de nature globale ont conduit à des changements de l'espace social urbain. Dans le cadre de la théorie du processus d'hétérogénéisation socio-spatiale, la présente démarche empirique vise à documenter l'analyse de la ségrégation socio-spatiale de divers groupes de la population de Bâle-Ville. Il apparaît que la «modernité» sociétale, la situation sociale et l'hétérogénéité 
des groupes observés exercent une influence déterminante sur l'apparition du nouveau modèle spatial. Des aspects de la qualité de l'environnement résidentiel donnent un éclairage sur l'influence de l'espace, relatif aux processus de ségrégation sociale.

Dipl.-Geogr. Susanne Eder, Geographisches Institut der Universität Basel, Abteilung Humangeographie / Stadt- und Regionalforschung, Klingelbergstrasse 16, $\mathrm{CH}-4056$ Basel.

e-mail: susanne.eder@unibas.ch

Manuskripteingang/received/manuscrit entré le 26.6.2001

Annahme zum Druck/accepted for publication/accepté pour l'impression: 14.12.2001 